\title{
Effect of compressibility on the stability of a vortex sheet in an ideal
}

magnetofluid

S. Duhau, and J. Gratton

Citation: The Physics of Fluids 16, 150 (1973); doi: 10.1063/1.1694162

View online: https://doi.org/10.1063/1.1694162

View Table of Contents: https://aip.scitation.org/toc/pfl/16/1

Published by the American Institute of Physics

\section{ARTICLES YOU MAY BE INTERESTED IN}

Hydromagnetic Stability at a Fluid Velocity Discontinuity between Compressible Fluids

The Physics of Fluids 7, 499 (1964); https://doi.org/10.1063/1.1711229

Hydromagnetic Reflection and Refraction at a Fluid Velocity Discontinuity

The Physics of Fluids 6, 508 (1963); https://doi.org/10.1063/1.1706765

\section{PHYSICS TODAY} WHITEPAPERS
ADVANCED LIGHT CURE ADHESIVES

READ NOW

Take a closer look at what these environmentally friendly adhesive systems can do
PRESENTED BY

(8) MASTERBOND 


\title{
Research Notes
}

Research Notes published in this Section include important research results of a preliminary nature which are of special interest to the physics of fuids and nere research contributions modifying results already published in the scientific literature. Research Notes cannot exceed five printed columns in length including space allowed for title, absiract, figures, tables, and references. The abstract should have three printed lines. Authors must shorten galley proofs of Research Notes longer than five printed columns before publication.

\section{Effect of compressibility on the stability of a vortex sheet in an ideal magnetofluid}

\author{
S. Duhau and J. Gratton
}

Departamento de Fisica, Facultad de Ciencias Exactas y Naturales, Universidad de Buenos Aires, Buenos Aires, Argentina

(Received 1 May 1972)

\begin{abstract}
The hydromagnetic stability of a vortex sheet with respect to all modulations is examined. A domain of absolute stability not previously noted is found for high compressibility. Stability criteria are given. Onset of instability is related to radiation of hydromagnetic waves.
\end{abstract}

The hydromagnetic Kelvin-Helmholtz problem has been investigated by several authors, ${ }^{1-3}$ and some applications to the interaction between the solar wind and the earth's magnetosphere have been attempted..$^{3-5}$ Recently, satellite observations have provided evidence of Kelvin-Helmholtz instability in the solar windmagnetosphere boundary, as discussed by Dungey and Southwood. ${ }^{6}$

Notwithstanding the fact that this problem has been widely studied, some important features that deserve further discussion have so far escaped attention. It is generally believed that the flow is unstable for relative velocities greater than a certain critical value which decreases as the compressibility is increased. ${ }^{1}$ We shall presently show that the effect of compressibility is more complicated. Indeed, when the compressibility is high enough, so that the sound velocity is smaller than the Alfvén velocity, we find two intervals of values of the streaming velocity in which the flow is stable with respect to all modes.

Let us consider two semi-infinite, compressible, perfectly conducting, inviscid fluids, separated by a vortex sheet that lies in the $(x, z)$ plane. The fluids are flowing along a uniform magnetic field $\mathbf{B}$, with velocities $u c_{A}$ $(y>0)$ and $-w_{A}(y<0)\left(c_{A}{ }^{2}=B^{2} / 4 \pi \rho\right)$. The $x$ axis will be taken in the direction of the field. The density $\rho$ and the pressure $p$ are assumed to be uniform in all space.

Any small arbitrary perturbation of the system can be expressed in terms of Fourier components of the form

$$
Q(y) \exp \left(-i \omega t+i k_{||} x+i k_{\perp} z\right) \text {. }
$$

Here, $k_{1 \mid}$ and $k_{\perp}$ are the parallel and normal components (with respect to $B$ ) of the wavenumber which characterizes the modulation of the interface, and $\omega$ is the frequency. We shall take

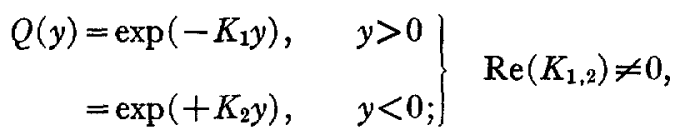

in order to ensure boundedness. When $K_{1,2}$ are purely imaginary, their signs in (1) will be chosen according to radiation criteria. $K_{1}$ and $K_{2}$ are determined by the dispersion relation of the slow and fast hydromagnetic waves:

$$
K_{1,2}{ }^{2}=k_{\perp}^{2}-k_{\|\|^{2}} \frac{\left(v_{1,2}^{2}-1\right)\left(v_{1,2^{2}}-c^{2}\right)}{\left(1+c^{2}\right)\left(v_{1,2}^{2}-s^{2}\right)} .
$$

Here,

$$
c=\left(\gamma p / \rho c_{A}^{2}\right)^{1 / 2}, \quad s=c /\left(1+c^{2}\right)^{1 / 2},
$$

are (in units of $c_{A}$ ) the velocity of sound, and the limiting value of the parallel component of the phase velocity of the slow wave for propagation almost perpendicular to the field. Also

$$
v_{1,2}=v \pm u, \quad v=\omega / k_{\|} c_{A},
$$

denote the parallel component of the phase velocity of the perturbation with respect to the media and to the observer. The dispersion relation for the oscillations of the interface is ${ }^{1}$ then

$$
\left[\left(v_{1}^{2}-1\right) / K_{1}\right] \pm\left[\left(v_{2}^{2}-1\right) / K_{2}\right]=0 .
$$

In Eq. (3), the + sign must be taken if $\operatorname{Re}\left(K_{1,2}\right) \neq 0$. Otherwise, the sign must be chosen according to radiation conditions.

When Eq. (3) is rationalized, one obtains [after 
factoring out the stable trivial roots $\left.(v u)^{2}=0\right]$ a fourthdegree polynomial in $v^{2}$ :

$$
P\left(v^{2}, u^{2}, q^{2}\right)=0, \quad q=\left(1+c^{2}\right) k_{\perp} / k_{\|} .
$$

The values of $u$ corresponding to marginal stability must be associated with the double root of Eq. (4). [Not all roots of Eq. (4) are roots of the dispersion relation (3), since the rationalization process introduces spurious roots, so that the solutions of Eq. (4) must be checked for consistency with Eq. (3).] These critical values of $u$ will depend on $q$, that is, on the obliqueness of the modulation of the interface. Therefore, in order to find the domains of absolute stability, that is, stability to all modes, one must look for the extremes of the critical values. These are determined by the conditions

$$
P=0, \quad \frac{\partial P}{\partial v}=0, \quad \frac{\partial P}{\partial q}=0 .
$$

The system (5) can be solved exactly for $u, v, q$. For brevity, we omit details of the analysis. One finds that minimum values of $u$ giving rise to double roots occur for $q=0$. These are

$$
u=u_{1}=\frac{1}{2}(1+s), \quad v=\frac{1}{2}(1-s),
$$

and

$$
u=u_{0}=1, \quad v=0 .
$$

It can easily be shown by expanding the dispersion relation in series that for a slightly oblique modulation $\left(q^{2} \ll 1\right)$ the double root (6a) leads to an overstability (v complex) of the system for $u>u_{1}$, and that the double root (6b) lends to a monotonic instability ( $v$ purely imaginary) for $u>1$ when $c<1$, and for $u<1$ when $c>1$. In passing, we mention that besides $u_{1}$ and $u_{0}$, there are, for $c>1$, other significant solutions of the system (5) which occur for $q=0$. They are related to other unstable modes. However, these other extreme values of $u$ are always greater than $u_{1}$. We shall not bother with them, since we are concerned only in finding the domains of stability with respect to all modes.

There is also a maximum value of $u$, given by

$$
u^{2}=u_{2}^{2}=\frac{1}{2}(1+s \sqrt{2}) \text {, }
$$

for

$$
q^{2}=\left[\left(1-c^{4}\right) / c^{4}\right]\left(c^{2}+s \sqrt{2}\right),
$$

corresponding to the double root

$$
v^{2}=\frac{1}{2}(1-s \sqrt{2}) \text {. }
$$

This is the only extreme which occurs for $q \neq 0$.

Besides, one can readily verify that in the special case $u=1$ ( $q$ arbitrary) the polynomial (4) always has a zero root. Then, by straightforward, but tedious, algebra, it is possible to show that the remaining third degree equation always has, provided $c<1$, three different positive roots $v^{2}>0$.

From the above considerations one concludes that:

(i) The system will be absolutely stable (within the model) for

$$
u<u_{1},
$$

in agreement with the result obtained by Fejer. ${ }^{1}$

(ii) The system will be also stable with respect to all modes provided

$$
c<1, \quad u_{2}<u<u_{0} .
$$

(iii) When $u$ and $c$ do not satisfy conditions (8) and (9), the system is unstable with respect to modes corresponding to some finite interval of $q$.

The present results are illustrated in Figs. 1 and 2.

The fact that the system is stable in the range (9) of sub-Alfvénic velocities when the compressibility is high was not hitherto recognized. On the contrary, other workers ${ }^{1,3}$ apparently deny that the system might be stable (to all modes) for any relative velocity greater than $2 u_{1}$.

We would like to point out that the critical relative velocities $2 u_{1}, 2 u_{0}$ that lead to the onset of instability for almost parallel modulation are just the minimum relative velocities required for the radiation of a pair of hydromagnetic waves from the interface. When $u=u_{1}$, $c<1$, the pair consists of a fast hydromagnetic wave

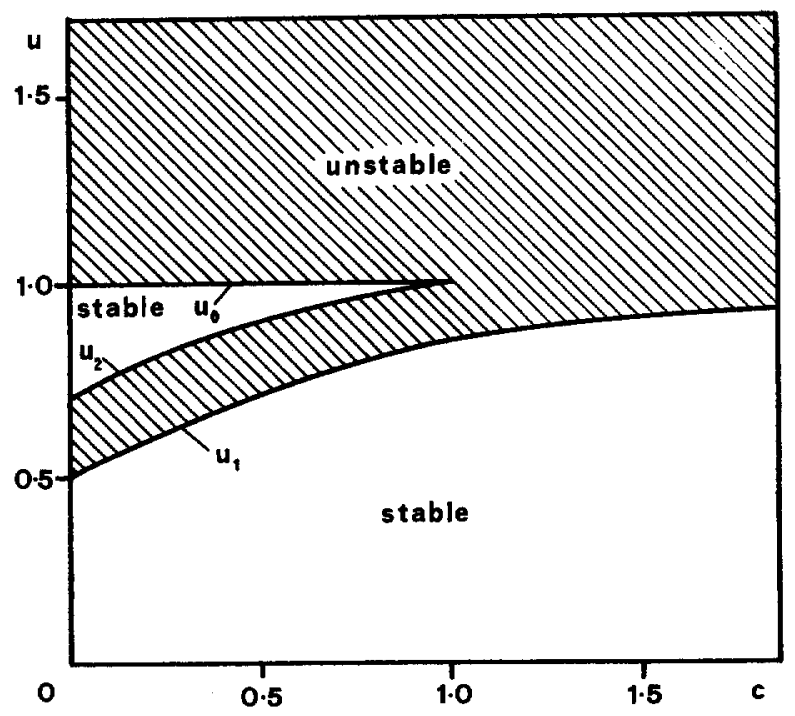

FIG. 1. Domains of stability with respect to all modes. The shaded regions correspond to situations in which the system is unstable. 


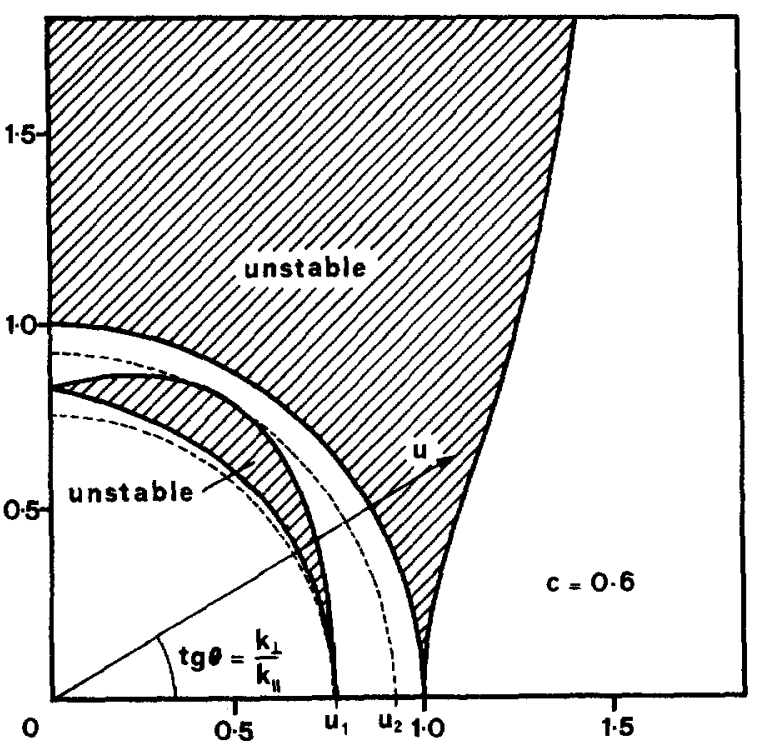

FIG. 2. Stability of the modes for $c=0.6$. The diagram is a polar plot of $u$ vs $\theta$, the angle which gives the direction of the modulation of the interface. The system is absolutely stable in the intervals $u<u_{1}$ and $u_{2}<u<u_{0}$.

traveling along the field lines in the half-space $y<0$, and a slow wave nearly perpendicular to the interface for $y>0$. When $u=u_{1}$, but $c>1$, both are slow waves; that in $y>0$ is almost normal to the interface while the other propagates parallel to the magnetic field. When $u=u_{0}, c<1$, both are fast hydromagnetic waves and travel along the field. ${ }^{7}$ In each case one of the waves (the one in $y>0$ ) carries negative energy. (All the present considerations are for positive $v$; if $v<0$ the situation is similar except for an exchange in the roles of both half-spaces.)

The radiation of waves from the boundary between two media in relative motion has been noted by several authors, ${ }^{8,9}$ and is one of the usual features of the Kelvin-Helmholtz problem. ${ }^{5,10}$ It has been shown ${ }^{10,11}$ that the radiation of waves and the Kelvin-Helmboltz instability are closely related.

It is our belief that this relationship will be useful in the search for stability criteria in more complicated problems of the same family.

This research was supported by the Comision Nacional de Estudios Geo-Heliofísicos and by the University of Buenos Aires under a grant from the Fondo Especial para la Investigación Científica.

1 J. A. Fejer, Phys. Fluids 7, 499 (1964).

2 A. K. Sen, Phys. Fluids 7, 1293 (1964).

${ }^{3}$ D. J. Southwood, Planet. Space Sci. 16, 587 (1968).

A. K. Sen. Planet. Space Sci. 13, 131 (1965).

J J. F. McKenzie, Planet. Space Sci. 18, 1 (1970).

J. W. Dungey and D. J. Southwood, Space Sci. Rev. 10, 672 (1970).

${ }^{7}$ Radiation of a pair of slow waves, both almost normal to the interface, occurs for $2 u>s$. This situation corresponds to the trivial zero root of Eq. (3) and, of course, does not lead to instability.

${ }^{8}$ J. W. Miles, J. Acoust. Soc. Am. 29, 226 (1957).

J. A. Fejer, Phys. Fluids 6, 508 (1963).

${ }^{10}$ See for example F. Gratton, J. Gratton, and J. Sánchez, Nucl. Fusion 11, 25 (1971); J. Gratton and F. Gratton, Plasma Phys. 13, 567 (1971); S. Duhau, F. Gratton, and J. Gratton, Phys. Fluids 14, $2067^{\prime}(1971)$.

${ }^{11}$ F. Gratton, and J. Gratton, Bull. Am. Phys. Soc. 17, 495 (1972).

\title{
Pressure limitation in a simple model of a tokamak
}

\author{
F. A. Haas and C. Ll. Thomas \\ United Kingdom Atomic Energy Authority, Research Group, Culham Laboratory, Abingdon, Berkshire, England
}

(Received 10 April 1972; final manuscript received 17 July 1972)

The simple model of a tokamak studied by Strauss is reinvestigated. By defining poloidal $\beta$ to be the ratio of the integrated pressure to the square of the toroidal current, it is shown that this quantity is bounded.

As a result of the encouraging studies made on tokamaks ${ }^{1,2}$ it is of interest to determine the theoretical upper limit (if any) to the pressure which may be confined. More precisely, we require the evaluation of the limit on the poloidal $\beta, \beta_{I}$. This is usually defined to $\mathrm{be}^{3}$

$$
\beta_{I}=8 \pi I^{-2} \int p d S,
$$

where $I$ is the toroidal component of current and the integral is taken over the minor cross-sectional area of the plasma.

Most studies of magnetohydrodynamic equilibria in tokamaks have been made in terms of the inverse aspect ratio $\epsilon$, where $\epsilon \ll 1$. For $\beta_{I} \sim 1,{ }^{3-5}$ the analysis can be carried through for an arbitrary pressure distribution. 'To investigate systems with $\beta_{T} \sim \epsilon^{-1}$, it is necessary to prescribe simple models for the pressure and toroidal current distribution. ${ }^{6,7,8} \mathrm{~A}$ feature common to all this work is that the confined pressure is limited by the appearance of a second magnetic axis, the upper limit to $\beta_{I}$ being

$$
\beta_{I}=A \epsilon^{-1},
$$

where $A$ is a number of order one and depends on the precise forms of pressure and current distribution, as well as on the shape of the plasma cross section. ${ }^{9}$ 